\title{
A Weighted Estimation for Risk Model
}

\author{
Mei Ling Huang ${ }^{1}$ and Ke Zhao ${ }^{2}$ \\ ${ }^{1}$ Department of Mathematics, Brock University, St. Catharines, ON, Canada L2S 3A1 \\ ${ }^{2}$ Alberta Health, Edmonton, AB, Canada T5J 4R7
}

Correspondence should be addressed to Mei Ling Huang; mhuang@brocku.ca

Received 16 June 2013; Accepted 19 August 2013

Academic Editors: J. Abellan, P. Dai Pra, and S. Sagitov

Copyright (C) 2013 M. L. Huang and K. Zhao. This is an open access article distributed under the Creative Commons Attribution License, which permits unrestricted use, distribution, and reproduction in any medium, provided the original work is properly cited.

\begin{abstract}
We propose a weighted estimation method for risk models. Two examples of natural disasters are studied: hurricane loss in the USA and forest fire loss in Canada. Risk data is often fitted by a heavy-tailed distribution, for example, a Pareto distribution, which has many applications in economics, actuarial science, survival analysis, networks, and other stochastic models. There is a difficulty in the inference of the Pareto distribution which has infinite moments in the heavy-tailed case. Firstly this paper applies the truncated Pareto distribution to overcome this difficulty. Secondly, we propose a weighted semiparametric method to estimate the truncated Pareto distribution. The idea of the new method is to place less weight on the extreme data values. This paper gives an exact efficiency function, $L_{1}$-optimal weights and $L_{2}$-optimal weights of the new estimator. Monte Carlo simulation results confirm the theoretical conclusions. The two above mentioned examples are analyzed by using the proposed method. This paper shows that the new estimation method is more efficient by mean square error relative to several existing methods and fits risk data well.
\end{abstract}

\section{Introduction}

1.1. Two Motivating Examples. In the recent years, many extreme events have occurred in financial markets, natural disasters, disease control, and industrial quality control. Natural disasters, for example, earthquakes, hurricanes, forest fires, volcanoes, and floods affect human life. It is important to predict and prepare for the next disaster occurrence and to estimate losses to inhabitants, insurance companies, and governments. In this section, we study two examples.

1.1.1. A Hurricane Loss Example. Strong winds, heavy rainfall, and storm surges caused by hurricanes cause death and destroy properties. They generate great losses to insurance companies as well. Figure 1 shows the 49 costliest Atlantic hurricane losses for the United States during 1900-2005 [1]. The measurement of this hurricane loss data is in US dollars; all dollars have been adjusted by using the inflation rates from 1900 to 2005.

From the data in [1], we note that the most costly hurricane is the 1926 Great Miami Hurricane with cost of damage of 157 billion which is 1.58 times larger than the second worst hurricane, the 1900 Galveston Hurricane. After
1926, on August 28, 2005 Hurricane Katrina caused damage of 81 billion. This is approximately 1.19 times larger than Hurricane Andrew in August 1992, which caused damage of 56 billion. On the other hand, we note that $80 \%$ of the hurricane losses are less than 21 billion. Many smaller hurricanes are not listed. These considerations raise a number of questions.

(a) How do we predict the loss of the next hurricane? Will it again be 1.58 times larger than the worst one so far?

(b) Do we keep the traditional approach which is to prepare for the worst event? Should we focus on the extreme events?

(c) What is the value at risk with $5 \%$ probability?

(d) How do we set an upper limit of the losses?

(e) How do we estimate the distribution of largest losses?

The objective of this study is to find the best model to fit the empirical data and to answer the above questions as accurately as possible. Since the data has large losses, we consider that the data should fit a heavy-tailed distribution. Studies on Pareto-type heavy-tailed distributions are rapidly 


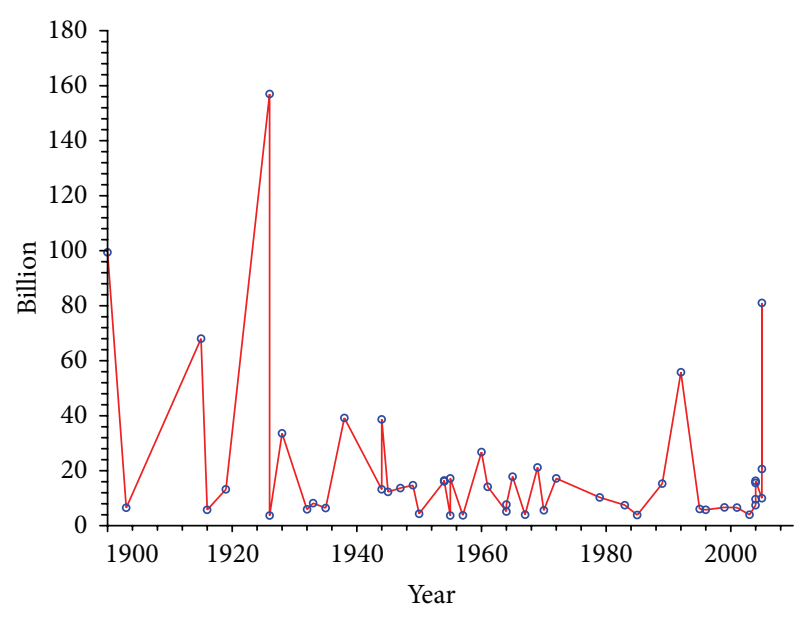

FIGURE 1: The 49 USA atlantic hurricane loss, 1900-2005. increasing with applications to extreme values, insurance, survival analysis, networks, and risk analysis [2,3]. Therefore, we choose a Pareto model. In this paper, we will propose a weighted method to study the hurricane loss data. The statistical analysis results are given in Section 6.

1.1.2. A Forest Fire Loss Example. Large forest fires have a significant impact on natural, social, and economic systems. However, most fires are extinguished in the initial stages and thus remain small. These smaller fires have a large probability of occurrence, but the resulting damage is almost negligible on an individual basis. Large forest fires, however, have a low probability of occurrence, but the damage and the losses are huge. So modelling large fire losses is becoming critical in the analysis of the risk of the next large forest fire. Figure 2 shows 30 forest fire losses during 1977 to 2006 in $A B$, Canada. We are concerned that the losses have been increasing over the last 30 years. The data listed in Table 1 are fire occurrence records from the Forestry Service (Council of Canadian Fire Marshals and Fire Commissioners, 2008, Canadian Fire Statistics, (http://www.ccfmfc.ca/stats.html). The database includes forest fire records for all the insurance losses. The data contains a relatively substantial number of large losses which convince us to use a Pareto model. The statistical analysis results are given in Section 6.

1.2. The Truncated Pareto Distribution. Many risk models with heavy tails have been developed using the class of the Pareto distributions. Some of these cases are found in city population sizes, the occurrence of natural resources, the sizes of firms, and personal income [3]. The Pareto distribution is on the list of distributions of Frechet domain which belongs to the extreme value distribution of type II [4]. It is important to explore estimation methods for Pareto distribution. There are theoretical difficulties in studying the Pareto distribution since it has infinite moments in heavytailed situations. We propose using the truncated Pareto distribution in these models to overcome these difficulties. Usually, we choose the upper limit as the largest value in the data set. In the recent years, the truncated Pareto distribution

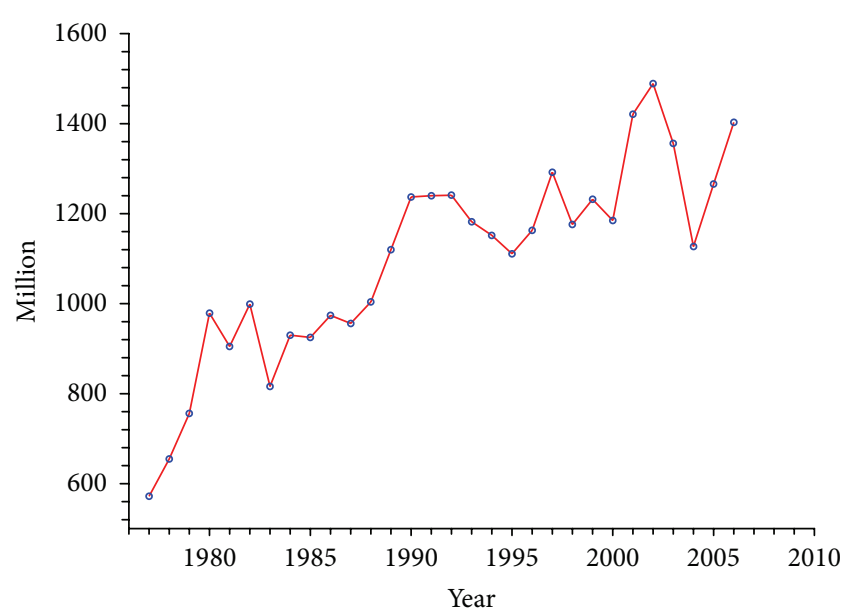

Figure 2: The 30 forest fire losses, 1977-2006, AB, Canada.

has become an alternative model for the original Pareto distribution.

There are several kinds of Pareto distributions. We consider a type I Pareto distribution in this paper [3].

Definition 1. The probability density function (p.d.f.) and the cumulative distribution function (c.d.f.) of a random variable $X$ having the Pareto distribution are given by

$$
\begin{gathered}
f_{p}(x)=\frac{\alpha \gamma^{\alpha}}{x^{(\alpha+1)}}, \quad 0<\gamma \leq x<\infty, \alpha>0, \\
F_{p}(x)=1-\left(\frac{\gamma}{x}\right)^{\alpha}, \quad 0<\gamma \leq x<\infty, \alpha>0,
\end{gathered}
$$

where $\alpha$ is the shape parameter.

When $0<\alpha \leq 1$, which is a heavy-tailed case, the mean and variance of $X$ are infinite, and the distribution is heavier on the right tail as $\alpha$ decreases.

The truncated Pareto distribution was originally used to describe the distribution of oil fields by size. It has a lower limit $\gamma$, an upper limit $\nu$, and a shape parameter $\alpha$. In fact, it has been shown that the truncated Pareto distribution fits better than the nontruncated distribution for positively skewed populations [5].

Definition 2. The p.d.f. and c.d.f. of a random variable $X$ having the truncated Pareto distribution are given by

$$
\begin{gathered}
f(x)=\frac{\alpha \gamma^{\alpha} x^{-\alpha-1}}{1-(\gamma / \nu)^{\alpha}}, \quad 0<\gamma \leq x \leq \nu<\infty, \alpha>0, \\
F(x)=1-\frac{\gamma^{\alpha}\left(x^{-\alpha}-\nu^{-\alpha}\right),}{1-(\gamma / \nu)^{\alpha}}, \quad 0<\gamma \leq x \leq \nu<\infty, \alpha>0 .
\end{gathered}
$$


TABLE 1: Forest fire loss data, AB, Canada (in million, 2006 CAD).

\begin{tabular}{lc}
\hline Year & Loss \\
\hline 1977 & 571.7 \\
1978 & 654.9 \\
1979 & 756.4 \\
1980 & 979.0 \\
1981 & 905.4 \\
1982 & 998.9 \\
1983 & 816.0 \\
1984 & 929.5 \\
1985 & 926.0 \\
1986 & 973.5 \\
1987 & 956.2 \\
1988 & $1,004.3$ \\
1989 & $1,119.6$ \\
1990 & $1,237.4$ \\
1991 & $1,239.7$ \\
1992 & $1,241.4$ \\
1993 & $1,181.9$ \\
1994 & $1,151.5$ \\
1995 & $1,110.8$ \\
1996 & $1,163.3$ \\
1997 & $1,291.6$ \\
1998 & $1,175.6$ \\
1999 & $1,231.9$ \\
2000 & $1,185.2$ \\
2001 & $1,420.8$ \\
2002 & $1,489.0$ \\
2003 & $1,356.1$ \\
2005 & $1,126.6$ \\
\hline & $1,265.9$ \\
1006 & $1,403.3$ \\
\hline
\end{tabular}

The quantile function of the truncated Pareto distribution is

$$
\begin{aligned}
F^{-1}(u)= & \left(\frac{1-u}{\gamma^{\alpha}}+\frac{u}{\nu^{\alpha}}\right)^{-1 / \alpha}, \\
& 0 \leq u \leq 1,0<\gamma \leq \nu<\infty, \quad \alpha>0 .
\end{aligned}
$$

The mean and variance of $X$ are

$$
\begin{array}{r}
\mu=\frac{\alpha \gamma^{\alpha}\left(\gamma^{1-\alpha}-\nu^{1-\alpha}\right)}{(\alpha-1)\left(1-(\gamma / \nu)^{\alpha}\right)}, \quad 0<\gamma<\nu<\infty, \alpha>0, \\
\sigma^{2}=\frac{\alpha \gamma^{\alpha}\left(\nu^{2-\alpha}-\gamma^{2-\alpha}\right)}{(2-\alpha)\left(1-(\gamma / \nu)^{\alpha}\right)^{2}}-\frac{\alpha^{2} \gamma^{2 \alpha}\left(\nu^{1-\alpha}-\gamma^{1-\alpha}\right)^{2}}{(1-\alpha)^{2}\left(1-(\gamma / \nu)^{\alpha}\right)^{2}}, \\
0<\gamma<\nu<\infty, \alpha>0 .
\end{array}
$$

1.3. The Weighted Empirical Distribution Function. Recently, some parametric estimation methods for the truncated Pareto distribution has been developed [6]. But there is an efficiency problem in the estimates of the distribution tails. The distribution tail values and their probabilities are important in many fields, for example, value at risk in risk analysis, survival probability in survival analysis, tolerance limits in quality control, prediction intervals, and confidence intervals. Classical statistical inference theory depends on the classical empirical distribution function (EDF) $S_{n}(x)$ :

$$
S_{n}(x)=\frac{1}{n} \sum_{i=1}^{n} I_{(-\infty, x]}\left(X_{i}\right), \quad \text { where } I_{A}= \begin{cases}1, & \text { if } x \in A ; \\ 0, & \text { if } x \notin A,\end{cases}
$$

$S_{n}(x)$ is a minimum variance unbiased estimator for the c.d.f. $F(x)$ based on a random sample $X_{1}, X_{2}, \ldots, X_{n}$. Note that $S_{n}(x)$ uses the equal weight $1 / n$ for each sample point. Should we use equal weights on extreme data values as well? Recently, authors have applied various weights to data points by using different philosophies; that is, the Jackknife method gives zero weight for eliminated data [7]; weighted bootstrap [8] and weighted empirical distribution functions or processes have been discussed $[9,10]$. But there are some difficulties to determine what weights should be used for the data points. Huang and Brill [11] introduced a weighted level crossing estimation method from a geometric point of view to visualize random samples in the $L_{1}$-optimal sense; the method improves the efficiency of the estimation of tails.

This paper proposes a semiparametric approach to estimate $F(x)$ in (4) using $L_{1}$-optimal and $L_{2}$-optimal weights. Both theoretical and simulation efficiencies are consistently improved when compared with existing methods. This method is based on a symmetric weighted empirical distribution function (SWEDF) of Huang [12], namely,

$$
\widehat{F_{n}^{*}}(x)=\sum_{i=1}^{n} I_{(-\infty, x]}\left(X_{(i)}\right) p_{n, i}, \quad x \in \Re, n>2,
$$

where the $p_{n, i}$ are symmetric general weights

$$
p_{n, i} \equiv \begin{cases}w, & i=2, \ldots, n-1,0<w<\frac{1}{n-2}, \\ w_{1, n} \equiv \frac{1}{2}(1-(n-2) w), & i=1, n,\end{cases}
$$

and $X_{(1)} \leq X_{(2)} \leq \cdots \leq X_{(n)}$ are the order statistics of the random sample. Note that

$$
0<p_{n, i}<1, \quad i=1, \ldots, n ; \quad \sum_{i=1}^{n} p_{n, i}=1 .
$$

The parameter $w$ in (9) is the weight for the middle $n-2$ data; $w_{1, n}$ in (9) is the weight for the extreme data. It is interesting to explore how the value of $w$ affects the estimation of a heavy-tailed distribution. We may use $w_{1, n}$ flexibly.

In Section 2, we propose a weighted method for estimating the shape parameter and the mean of the truncated Pareto distribution. In Section 3, an exact efficiency function of the new mean estimator relative to the classical estimator is derived. Section 4 explores the $L_{1}$-optimal weights and $L_{2}$-optimal weights for estimating the mean. Section 5 gives 
results of Monte Carlo simulations. The simulation efficiencies are consistent with the exact efficiencies in Section 3. In Section 6, we analyze the hurricane loss data and forest fire loss data given in Section 1 by using the proposed method. The statistical inference in these examples shows that the estimated distribution curve by using proposed weighted estimation method fits the tails of data better, relative to several existing methods. Suggestions for further studies are also discussed.

\section{Estimation Methods}

In this section, we discuss the existing and proposed methodologies for the truncated Pareto distribution. Consider a random sample $X_{1}, X_{2}, \ldots, X_{n}$ from the distribution in (3), and let $X_{(1)} \leq X_{(2)} \leq \cdots \leq X_{(n)}$ denote its order statistics.

2.1. Maximum Likelihood Estimators (Hill, Beg, and Aban). There are several different maximum likelihood estimators (MLE) for estimating the shape parameter $\alpha$.

A popular estimator is the Hill [13] MLE, which uses the $r$ largest order statistics, $1 \leq r \leq n-1$, to estimate the original Pareto shape parameter $\alpha$ in (1). When applying it to the truncated Pareto distribution in (3), it is defined as

$$
\widehat{\alpha}_{\text {Hill }}=\left[r^{-1} \sum_{i=1}^{r}\left\{\ln X_{(n-i+1)}-\ln X_{(n-r)}\right\}\right]^{-1} .
$$

Beg [5] developed the MLE method for the truncated Pareto distribution when $\nu$ is known. The Beg MLE for $\alpha$ can be obtained by solving the following equation:

$$
n\left[\frac{1}{\widehat{\alpha}_{\mathrm{Beg}}}+\frac{\ln \left(X_{(1)} / \widehat{\alpha}_{\mathrm{Beg}}\right)}{\left(\left(X_{(1)} / \nu\right)^{-\widehat{\alpha}_{\mathrm{Beg}}}-1\right)}-\ln \left(\frac{g}{X_{(1)}}\right)\right]=0,
$$

where $X_{(1)}=\min \left(X_{1}, X_{2}, \ldots, X_{n}\right)$ and $g=\left(X_{(1)} X_{(2)}\right.$ $\left.\cdots X_{(n)}\right)^{1 / n}$ is the sample geometric mean.

Aban's MLE [6] when $\gamma, v$ are known is obtained by solving the equation

$$
\frac{n}{\widehat{\alpha}_{\text {Aban }}}+\frac{n(\gamma / \nu)^{\widehat{\alpha}_{\text {Aban }}} \ln (\gamma / \nu)}{1-(\gamma / \nu)^{\widehat{\alpha}_{\text {Aban }}}}-\sum_{i=1}^{n}\left[\ln X_{(i)}-\ln \gamma\right]=0,
$$

where $\gamma \leq X_{(i)} \leq \nu$ and $n$ is the sample size.

Note that we may use estimators $\widehat{\gamma}=\min \left(X_{1}, X_{2}, \ldots, X_{n}\right)$ and $\hat{v}=\max \left(X_{1}, X_{2}, \ldots, X_{n}\right)$ in (14) when $\gamma, v$ are unknown. A similar situation is in the following equations (16) and (18). There are other estimators of $\gamma$ and $\nu$ in the literature, for example, Cooke [14].

2.2. Moment Estimator. To estimate the population truncated Pareto mean, the sample mean estimator is

$$
\widehat{\mu}_{\bar{X}}=\bar{X}=\frac{1}{n} \sum_{i=1}^{n} X_{i}
$$

A Moment estimator $\widehat{\alpha}_{M}$ for estimating $\alpha$ is the solution of the equation

$$
\bar{X}=\frac{\widehat{\alpha}_{M} \gamma^{\widehat{\alpha}_{M}}\left(\gamma^{1-\widehat{\alpha}_{M}}-\nu^{1-\widehat{\alpha}_{M}}\right)}{\left(\widehat{\alpha}_{M}-1\right)\left(1-(\gamma / \nu)^{\widehat{\alpha}_{M}}\right)}, \quad \widehat{\alpha}_{M}>0
$$

2.3. A Proposed Weighted Estimator. Now, to estimate the population truncated Pareto mean, we define a weighted mean based on the weighted empirical distribution function in (9) as

$$
\begin{aligned}
\widehat{\mu}_{w} & =\bar{X}_{w}=\frac{1}{n} \sum_{i=1}^{n} p_{n, i} X_{(i)} \\
& =\sum_{i=2}^{n-1} w X_{(i)}+\frac{1}{2}(1-(n-2) w)\left[X_{(1)}+X_{(n)}\right],
\end{aligned}
$$

where $n$ is the sample size and $w$ is the weight as defined in (9). Then, for estimating $\alpha$, we define $\widehat{\alpha}_{w}$ to be the solution of the equation

$$
\bar{X}_{w}=\frac{\widehat{\alpha}_{w} \gamma^{\widehat{\alpha}_{w}}\left(\gamma^{1-\widehat{\alpha}_{w}}-\nu^{1-\widehat{\alpha}_{w}}\right)}{\left(\widehat{\alpha}_{w}-1\right)\left(1-(\gamma / \nu)^{\widehat{\alpha}_{w}}\right)}, \quad \widehat{\alpha}_{w}>0
$$

\section{An Exact Efficiency Function}

In this section, an exact efficiency function of the weighted estimator $\widehat{\mu}_{w}$ in (17) relative to the sample mean $\bar{X}$ in (15) is given. The mathematical derivations are given in the appendix.

Theorem 3. The mean and mean square error (MSE), of $\widehat{\mu}_{w}$ in (17) when $\alpha>0$, are given by

$$
\begin{aligned}
& E\left[\widehat{\mu}_{w}\right]=\gamma \sum_{i=2}^{n-1} \sum_{k=0}^{\infty}\left\{\left(\begin{array}{c}
\frac{1}{\alpha}+k-1 \\
k
\end{array}\right)\right. \\
& \times \rho^{k}\left[\frac{\Gamma(n+1) \Gamma(k+i)}{\Gamma(i) \Gamma(k+n+1)}\right. \\
& +\frac{1}{2}(1-(n-2) w) \\
& \left.\left.\times \rho^{k}\left(\frac{\Gamma(n+1) \Gamma(k+1)}{\Gamma(k+n+1)}+\frac{n}{k+n}\right)\right]\right\}, \\
& \operatorname{MSE}\left(\widehat{\mu}_{w}\right)=a w^{2}+b w+c, \quad \text { where } 0<w<1 \text {, } \\
& a=A+\frac{1}{4}(n-2)^{2} B-(n-2) C, \\
& b=-\frac{1}{2}(n-2) B+C-2 \mu D+\mu(n-2) E, \\
& c=\frac{1}{4} B-\mu E+\mu^{2}, \\
& A=\gamma \sum_{i=2}^{n-1} \sum_{k=0}^{\infty}\left(\begin{array}{c}
\frac{2}{\alpha}+k-1 \\
k
\end{array}\right) \rho^{k} \frac{\Gamma(n+1) \Gamma(k+i)}{\Gamma(i) \Gamma(k+n+1)}
\end{aligned}
$$




$$
\begin{aligned}
& +2 \gamma^{2} \sum_{i=2}^{n-2} \sum_{j=i+1}^{n-1} \sum_{k=0}^{\infty} \sum_{l=0}^{\infty}\left\{\left(\begin{array}{c}
\frac{1}{\alpha}+k-1 \\
k
\end{array}\right)\left(\begin{array}{c}
\frac{1}{\alpha}+l-1 \\
l
\end{array}\right)\right. \\
& \times \rho^{k+l} \\
& \left.\times\left[\frac{\Gamma(n+1) \Gamma(k+i) \Gamma(k+l+j)}{\Gamma(i) \Gamma(k+j) \Gamma(k+l+n+1)}\right]\right\}, \\
& B=\gamma \sum_{k=0}^{\infty}\left\{\left(\begin{array}{c}
\frac{2}{\alpha}+k-1 \\
k
\end{array}\right)\right. \\
& \operatorname{EFF}\left(\widehat{\mu}_{w}\right) \\
& =\frac{\operatorname{Var}(\bar{X})}{\operatorname{MSE}\left(\widehat{\mu}_{w}\right)}=\frac{\sigma^{2} / n}{\operatorname{MSE}\left(\widehat{\mu}_{w}\right)} \\
& =\frac{\alpha \gamma^{\alpha}\left(\nu^{2-\alpha}-\gamma^{2-\alpha}\right) /\left\{(2-\alpha)\left(1-(\gamma / \nu)^{\alpha}\right)\right\}}{n M S E\left(\hat{\mu}_{w}\right)}, \\
& -\frac{\alpha^{2} \gamma^{2 \alpha}\left(\nu^{1-\alpha}-\gamma^{1-\alpha}\right)^{2} /\left\{(1-\alpha)^{2}\left(1-(\gamma / \nu)^{\alpha}\right)^{2}\right\}}{n M S E\left(\hat{\mu}_{w}\right)},
\end{aligned}
$$$$
\left.\times \rho^{k}\left[\frac{\Gamma(n+1) \Gamma(k+1)}{\Gamma(k+n+1)}+\frac{n}{k+n}\right]\right\}
$$$$
+2 \gamma^{2} \sum_{k=0}^{\infty} \sum_{l=0}^{\infty}\left\{\left(\begin{array}{c}
\frac{1}{\alpha}+k-1 \\
k
\end{array}\right)\left(\begin{array}{c}
\frac{1}{\alpha}+l-1 \\
l
\end{array}\right)\right.
$$$$
\left.\times \rho^{k+l}\left[\frac{\Gamma(n+1) \Gamma(k+1)}{\Gamma(k+n)(k+l+n)}\right]\right\},
$$$$
C=\gamma^{2} \sum_{i=2}^{n-1} \sum_{k=0}^{\infty} \sum_{l=0}^{\infty}\left\{\left(\begin{array}{c}
\frac{1}{\alpha}+k-1 \\
k
\end{array}\right)\left(\begin{array}{c}
\frac{1}{\alpha}+l-1 \\
l
\end{array}\right)\right.
$$$$
\times \rho^{k+l}\left[\frac{\Gamma(n+1) \Gamma(k+1) \Gamma(k+l+i)}{\Gamma(k+i) \Gamma(k+l+n+1)}\right.
$$$$
\left.\left.+\frac{\Gamma(n+1) \Gamma(k+i)}{\Gamma(i) \Gamma(k+n)(k+l+n)}\right]\right\},
$$$$
D=\gamma \sum_{i=2}^{n-1} \sum_{k=0}^{\infty}\left\{\left(\begin{array}{c}
\frac{1}{\alpha}+k-1 \\
k
\end{array}\right)\right.
$$$$
\left.\times \rho^{k} \frac{\Gamma(n+1) \Gamma(k+i)}{\Gamma(i) \Gamma(k+n+1)}\right\},
$$$$
E=\gamma \sum_{k=0}^{\infty}\left\{\left(\begin{array}{c}
\frac{1}{\alpha}+k-1 \\
k
\end{array}\right)\right.
$$$$
\times \rho^{k}\left[\frac{\Gamma(n+1) \Gamma(k+1)}{\Gamma(k+n+1)}\right.
$$$$
\left.\left.+\frac{n}{k+n}\right]\right\}
$$

where $\rho=\left(g^{\alpha}-1\right) / g^{\alpha}, g=\nu / \gamma, 0<\rho<1,0<g<1$, $0<\gamma \leq x \leq v<\infty$,

$$
\Gamma(z)=\int_{0}^{\infty} t^{z-1} e^{-t} d t, \quad z>0, \quad \text { is the Gamma function. }
$$

where $\sigma^{2}$ and $\operatorname{MSE}\left(\widehat{\mu}_{w}\right)$ are given in (7) and (20).

\section{Optimal Weights}

4.1. $L_{1}$-Optimal Weights. Huang and Brill [11] proposed an $L_{1}$-optimal weight which is based on Manhattan metric for the $\widehat{F_{n}^{*}}(x)$ in (9). It is

$$
w_{L_{1} \text { opt }}=\frac{1}{\sqrt{n(n-1)}}>\frac{1}{n}, \quad \text { for the middle data. }
$$

Huang and Brill [11] proved that the exact efficiency (EFF) of $\widehat{F_{n}^{*}}(x)$ in (9) relative to the EDF $S_{n}(x)$ exceeds 1 on the tails of the distribution. In this paper, we use this $w_{L_{1} \text {-opt }}$ weight in order to improve the efficiency of estimating the tail probability of the truncated Pareto distribution. Huang [12] indicates that in general for any distribution if $w>1 / n$, which means putting more weight on the middle data, the efficiency of estimating the tail probability will be improved.

Next, we explore an alternative $L_{2}$-optimal weight.

\section{2. $L_{2}$-Optimal Weights}

Corollary 5. An $L_{2}$-optimal weight $w_{L_{2} \text {-opt }}$ for the efficiency function of the $\widehat{\mu}_{w}$ given in (17) for estimating the population mean in (3) relative to the sample mean $\bar{X}$ in (16) when $n>$ $4 C / B+2$ is given by

$$
w_{L_{2}-o p t}=-\frac{b}{2 a} .
$$

The minimum $M S E_{\min }\left(\widehat{\mu}_{w}\right)$ and $E F F_{\max }\left(\widehat{\mu}_{w}\right)$ are given by

$$
M S E_{\min }\left(\widehat{\mu}_{w}\right)=-\frac{b^{2}}{4 a}+c,
$$

$$
\begin{aligned}
E F F_{\max }\left(\hat{\mu}_{w}\right) & \\
= & \frac{\alpha \gamma^{\alpha}\left(\nu^{2-\alpha}-\gamma^{2-\alpha}\right) /\left\{(2-\alpha)\left(1-(\gamma / \nu)^{\alpha}\right)\right\}}{n\left(-b^{2} /(4 a)+c\right)} \\
& -\frac{\alpha^{2} \gamma^{2 \alpha}\left(\nu^{1-\alpha}-\gamma^{1-\alpha}\right)^{2} /\left\{(1-\alpha)^{2}\left(1-(\gamma / \nu)^{\alpha}\right)^{2}\right\}}{n\left(-b^{2} /(4 a)+c\right)} .
\end{aligned}
$$

In (24) and (25), $a, b, c, C$, and $B$ are defined in (20). 
TABLE 2: Exact efficiencies of $\widehat{\mu}_{w}$ relative to other estimators by using $L_{2}$-optimal weights, $\gamma=1, \nu=10$.

\begin{tabular}{|c|c|c|c|c|c|c|c|}
\hline Sample size & $\alpha$ & $w_{L_{2} \text {-opt }}$ & $w=1 / n$ & $w_{L_{1} \text {-opt }}$ & $\operatorname{MSE}\left(\widehat{\mu}_{w}\right)$ & $\operatorname{MSE}(\bar{X})$ & $\operatorname{EFF}\left(\widehat{\mu}_{w(\bar{X})}\right)$ \\
\hline \multirow{3}{*}{$n=10$} & 0.8 & 0.10396 & \multirow{3}{*}{0.1} & \multirow{3}{*}{0.10541} & 0.4007 & 0.4034 & 1.0068 \\
\hline & 1.8 & 0.11446 & & & 0.1406 & 0.1647 & 1.1717 \\
\hline & 3 & 0.11847 & & & 0.0365 & 0.0493 & 1.3501 \\
\hline \multirow{3}{*}{$n=20$} & 0.8 & 0.05015 & \multirow{3}{*}{0.05} & \multirow{3}{*}{0.05130} & 0.2017 & 0.2017 & 1.0001 \\
\hline & 1.8 & 0.05255 & & & 0.0766 & 0.0824 & 1.0759 \\
\hline & 3 & 0.05463 & & & 0.0217 & 0.0247 & 1.1367 \\
\hline \multirow{3}{*}{$n=30$} & 0.8 & 0.03323 & \multirow{3}{*}{0.03333} & \multirow{3}{*}{0.03390} & 0.1344 & 0.1345 & 1.0003 \\
\hline & 1.8 & 0.03425 & & & 0.0528 & 0.0549 & 1.0399 \\
\hline & 3 & 0.03529 & & & 0.0155 & 0.0164 & 1.0628 \\
\hline \multirow{3}{*}{$n=50$} & 0.8 & 0.02021 & \multirow{3}{*}{0.02} & \multirow{3}{*}{0.02020} & 0.0968 & 0.1063 & 1.0978 \\
\hline & 1.8 & 0.02159 & & & 0.0514 & 0.0592 & 1.1536 \\
\hline & 3 & 0.02298 & & & 0.0274 & 0.0352 & 1.2849 \\
\hline \multirow{3}{*}{$n=100$} & 0.8 & 0.01001 & \multirow{3}{*}{0.01} & \multirow{3}{*}{0.01005} & 0.0765 & 0.0830 & 1.0845 \\
\hline & 1.8 & 0.01002 & & & 0.0542 & 0.0576 & 1.0637 \\
\hline & 3 & 0.01002 & & & 0.0380 & 0.0400 & 1.0526 \\
\hline
\end{tabular}

The proof of Corollary 5 is in the appendix.

Table 2 lists the values of $w_{L_{2} \text {-opt }}, w_{L_{1} \text { opt }}, \operatorname{MSE}_{\text {min }}\left(\widehat{\mu}_{w}\right)$, $\operatorname{MSE}_{\text {min }}(\bar{X})$, and the exact $\operatorname{EFF}_{\text {max }}\left(\widehat{\mu}_{w}\right)$ of $\widehat{\mu}_{w}$ relative to $\bar{X}$ for $n=10,20,30,50$, and $100 ; \alpha=0.8,1.8$ and 3 , by using $(24)$ and (25). We note that all values of $w_{L_{2} \text {-opt }}$ are greater than $1 / n$, and all exact $\operatorname{EFF}_{\max }\left(\widehat{\mu}_{w}\right)$ relative to $\bar{X}$ are greater than 1 .

Remark 6 . The $w_{L_{1} \text {-opt }}$ in (23) is totally nonparametric; it is more robust and easy to use. Note that $w_{L_{2} \text {-opt }}$ in (24) depends on the parameter $\alpha$. In practice, we may estimate $\alpha$ first then obtain a $w_{L_{2} \text {-opt }}$, while still keeping the optimal advantage. Of course, we use the given $\alpha$ values in the simulations. However, $w_{L_{1} \text { opt }}$ and $w_{L_{2} \text { opt }}$ are close to each other when $n>100$.

\section{Simulations}

Next, we use simulations to compare the performance of the three parametric MLE estimators, that is, Hill's, Beg's, and Aban's estimators in (12), (13), and (14), and the two semiparametric estimators, that is, the Moment and the weighted estimators in (16) and (18), for estimating the shape parameter $\alpha$ in (3). We generate $m=1,000$ random samples of size $n=100$ from the distribution of (3). $w_{L_{1} \text {-opt }}$ is only used for the weighted estimator, since Table 2 indicates that the values of $w_{L_{2} \text {-opt }}$ and $w_{L_{1} \text {-opt }}$ are close to each other when $n=100$.

We know that if $\alpha \leq 2$, the original Pareto distribution has an infinite variance; if $\alpha \leq 1$, then the mean is infinite. These cases have inference difficulties. We focus on $\alpha=0.5,0.8$, and 1.8 , and let $\gamma=1, \nu=10$. Figure 3 contains the box-plots of the comparison of these five estimators. Note that in the cases $\alpha=0.5$ and 0.8, Beg's and Hill's estimators have large biases, and the weighted, Aban's, and the moment estimators performed very well; but sometimes, Aban's MLE estimates have unstable solutions. When $\alpha=1.8$, there are similar results except Hill's and Beg's estimators performed better. It is interesting to see that, in all three cases, the median of the weighted estimator for $\alpha$ is relatively larger than Aban's and the Moment's ones. We will discuss how these affect the tail estimation in the next section. The simulations were run by using MAPLE 15 with double precision.

\section{Examples}

Now we use the proposed method and compare it with existing methods to analyze the data of the two examples outlined in Section 1.

\subsection{Hurricane Loss Example}

6.1.1. Comparison of Four Estimation Methods. At first, We look at the hurricane loss example in Section 1.1.1. Based on the simulation results in Figure 3, we consider the three better estimators out of the five in the heavy-tailed case, that is, Aban's, Moment, and the weighted estimators in (27), (28), and (29) for the truncated Pareto p.d.f. $f(x)$ in (3) and the c.d.f. $F(x)$ in (4). We also compare them with Hill's estimator in (26) for the original Pareto p.d.f. $f_{p}(x)$ in (1) and the c.d.f. $F_{p}(x)$ in $(2)$. Here

$$
\widehat{f}_{\text {Hill }}(x)=\frac{\widehat{\alpha}_{\text {Hill }} \gamma^{\widehat{\alpha}_{\text {Hill }}}}{x^{\widehat{\alpha}_{w}}}, \quad \widehat{F}_{\text {Hill }}(x)=1-\left(\frac{\gamma}{x}\right)^{\widehat{\alpha}_{\text {Hill }}},
$$

where $\widehat{\alpha}_{\text {Hill }}$ is given in (12) with $r=n-1$; consider

$$
\begin{aligned}
& \widehat{f}_{\mathrm{MLE}}(x)=\frac{\widehat{\alpha}_{\mathrm{MLE}} \gamma^{\widehat{\alpha}_{\mathrm{MLE}}} x^{-\widehat{\alpha}_{\mathrm{MLE}}-1}}{1-(\gamma / \nu)^{\widehat{\alpha}_{\mathrm{MLE}}}}, \\
& \widehat{F}_{\mathrm{MLE}}(x)=1-\frac{\gamma^{\widehat{\alpha}_{\mathrm{MLE}}}\left(x^{-\widehat{\alpha}_{\mathrm{MLE}}}-\nu^{-\widehat{\alpha}_{\mathrm{MLE}}}\right)}{1-(\gamma / \nu)^{\widehat{\alpha}_{\mathrm{MLE}}}},
\end{aligned}
$$


TABLE 3: Estimation of index, mean, median, and VaR for hurricane loss data.

\begin{tabular}{lcccc}
\hline Method & $\widehat{\alpha}$ & $\widehat{\mu}$ & Median & $5 \%$ value at risk \\
\hline Pareto $_{\text {(Hill) }}$ & 0.8126 & $\infty$ & 8.68 billion & 147.68 billion \\
$\operatorname{TPD}_{(\text {Aban) }}$ & 0.6206 & 21.74 billion & 9.73 billion & 85.15 billion \\
$\operatorname{TPD}_{\text {(Moment) }}$ & 0.6476 & 20.48 billion & 9.47 billion & 82.55 billion \\
$\operatorname{TPD}_{\text {(Weighted) }}$ & 0.6990 & 19.37 billion & 9.02 billion & 77.58 billion \\
\hline
\end{tabular}
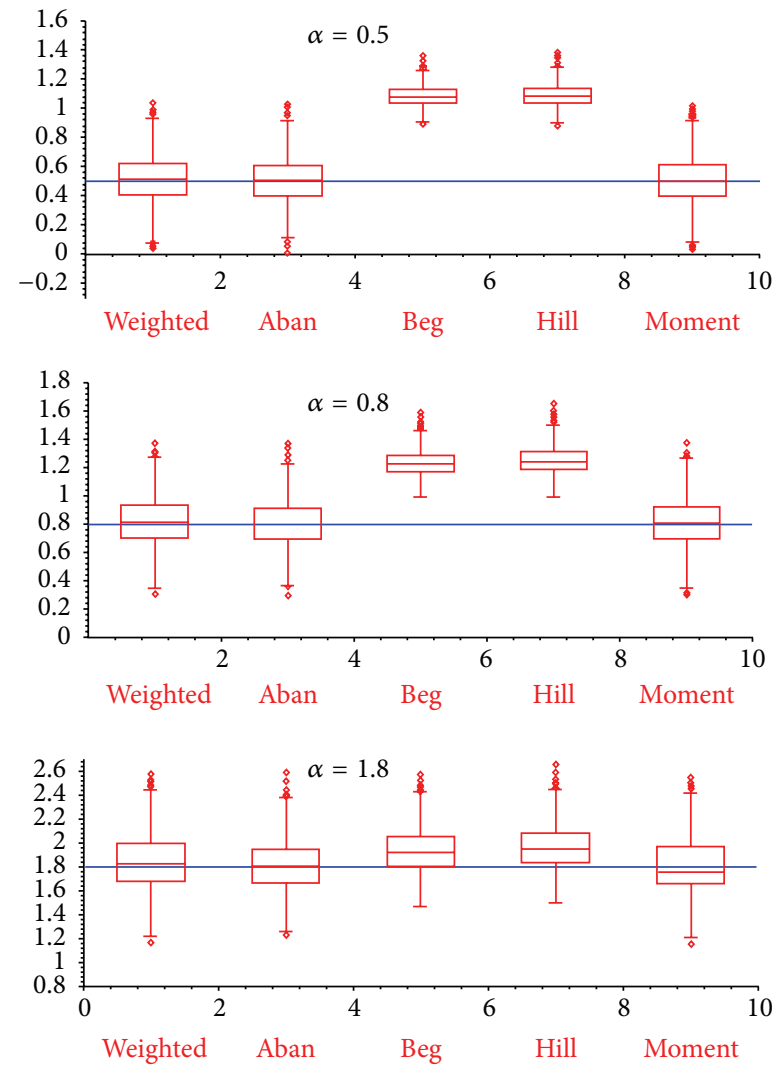

FIGURE 3: Box-plot of the estimators for the shape parameter $\alpha$, by using $L_{1}$-optimal weight, $\gamma=1, \nu=10$, sample size $n=100$, and generating times $m=1,000$.

where $\widehat{\alpha}_{\mathrm{MLE}}$ is Aban's MLE given in (14); consider

$$
\begin{aligned}
& \widehat{f}_{M}(x)=\frac{\widehat{\alpha}_{M} \gamma^{\widehat{\alpha}_{M}} x^{-\widehat{\alpha}_{M}-1}}{1-(\gamma / \nu)^{\widehat{\alpha}_{M}}}, \\
& \widehat{F}_{M}(x)=1-\frac{\gamma^{\widehat{\alpha}_{M}}\left(x^{-\widehat{\alpha}_{M}}-\nu^{-\widehat{\alpha}_{M}}\right)}{1-(\gamma / \nu)^{\widehat{\alpha}_{M}}},
\end{aligned}
$$

where $\widehat{\alpha}_{M}$ is given in (16); consider

$$
\widehat{f}_{w}(x)=\frac{\widehat{\alpha}_{w} \gamma^{\widehat{\alpha}_{w}} x^{-\widehat{\alpha}_{w}-1}}{1-(\gamma / \nu)^{\widehat{\alpha}_{w}}}, \quad \widehat{F}_{w}(x)=1-\frac{\gamma^{\widehat{\alpha}_{w}}\left(x^{-\widehat{\alpha}_{w}}-\nu^{-\widehat{\alpha}_{w}}\right)}{1-(\gamma / \nu)^{\widehat{\alpha}_{w}}},
$$

where $\widehat{\alpha}_{w}$ is given in (18), using the weight $w_{L_{1} \text {-opt }}=$ $1 / \sqrt{n(n-1)}$.
The results of these four methods are listed in Table 3 by using the hurricane loss data, where $\hat{\gamma}=X_{(\min )}, \widehat{v}=X_{(\max )}$, and $n=49$.

Figure 4 is a log-log plot showing the upper tail for the hurricane loss data. In this plot, the circles represent the real data, and the straight line represents the estimated original Pareto distribution. The dashed line, dotted line, and thick solid line represent the estimated truncated Pareto distributions by using Aban's, Moment, and Huang's estimators, respectively. We observe two conclusions intuitively.

(1) The original Pareto distribution (straight line) does not fit the data well in the tail. The truncated Pareto distribution fits the data very well using all three estimation methods (Aban, Moment, and weighted). Note that the $5 \%$ value at risk estimated by the original Pareto model is 147 billion; the three truncated model estimates are about 80 billion. It appears that the original Pareto model overestimates the risk. An insurance company would set a high premium if it is using the Pareto model, with the result that many people cannot afford to buy insurance.

(2) We examine the three truncated Pareto estimates. Around the tail, the weighted estimate fits the data the best; that is, the $\ln (P\{X>x\})$ curve turns downward more quickly following the trend of the data pattern because the weighted estimate $\widehat{\alpha}_{w}$ is largest among the three truncated model estimates (this is consistent with the simulation results in Figure 4), and the weighted mean estimate $\widehat{\mu}_{w}$ and the $5 \%$ value at risk are the smallest among the three methods (this is obtained by placing less weight on the extreme value, e.g., the 1926 great miami hurricane's loss), so its estimated distribution is less heavy in the tail compared with other methods.

Next, in order to confirm these conclusions, we run three goodness of-fit tests. Later, we define the absolute error and integrated error as the measures of the distance from the empirical data points to the estimated Pareto curve and truncated Pareto curves.

6.1.2. Goodness-of-Fit Tests. Our objective is to test if the estimated distributions in (26)-(29) fit the data properly. We test the hypotheses $H_{0}: F(x)=F^{*}(x)$ against $H_{1}$ : $F(x) \neq F^{*}(x)$, where $F(x)$ is the true unknown distribution function and $F^{*}(x)$ is the estimated Pareto c.d.f. in (26) or the estimated truncated Pareto c.d.f. in (27)-(29). In this paper we use three EDF goodness-of-fit tests. 


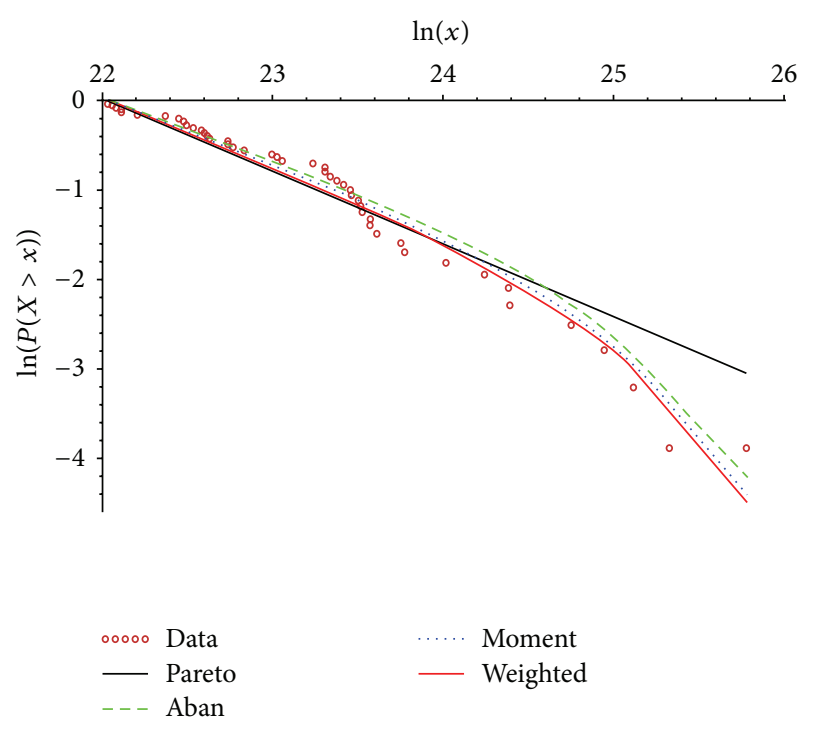

FIGURE 4: Log-log plot of the hurricane loss data.

(1) The Kolmogorov-Smirnov (K-S) test [15]: the test statistic is given by

$$
T=\sup _{x}\left|F^{*}(x)-S_{n}(x)\right|, \quad-\infty<x<\infty,
$$

where $S_{n}(x)$ is the EDF in (8). Under $H_{0}$, the two-tailed $P$ value for the K-S test is as follows:

$$
\begin{aligned}
& P \text {-value } \\
& \qquad=1-\left[1-T \sum_{j=0}^{[n(1-T)]} \frac{n !}{j !(n-j) !}\left(1-T-\frac{j}{n}\right)^{n-j}\left(t+\frac{j}{n}\right)^{j-1}\right]^{2},
\end{aligned}
$$

where $[n(1-T)]$ is the integer part of $n(1-T)$.

(2) Anderson and Darling [16] test (A-D test) introduced a measure of "distance" between the empirical distribution $S_{n}(x)$ and the proposed c.d.f. $F^{*}(x)$ by using a metric function space

$$
W_{n}^{2}=n \int_{-\infty}^{\infty}\left[S_{n}(x)-F^{*}(x)\right]^{2} \psi\left(F^{*}(x)\right) d F,
$$

where $\psi(u)=1 /(u /(1-u))$ is a weight function, with $u=$ $F(x)$. Let $u_{i}=F\left(X_{(i)}\right), i=1, \ldots, n$, and under $H_{0}$ the test statistic and $P$-value are given by

$$
\begin{gathered}
W_{n}^{2}=-n-\frac{1}{n} \sum_{j=1}^{n}(2 j-1)\left(\log \left(u_{j}\right)+\log \left(1-u_{n-j+1}\right)\right), \\
P \text {-value }=1-\frac{\sqrt{2}}{z} \sum_{j=0}^{\infty}\left\{\frac{(-1)^{j} \Gamma(j+1 / 2)(4 j+1)}{j !} e^{-(4 j+1)^{2} \pi^{2} /(8 z)}\right. \\
\left.\times \int_{0}^{\infty} e^{\left((z / 8) /\left(w^{2}+1\right)-(4 j+1)^{2} \pi^{2} w^{2} /(8 z)\right)} d w\right\},
\end{gathered}
$$

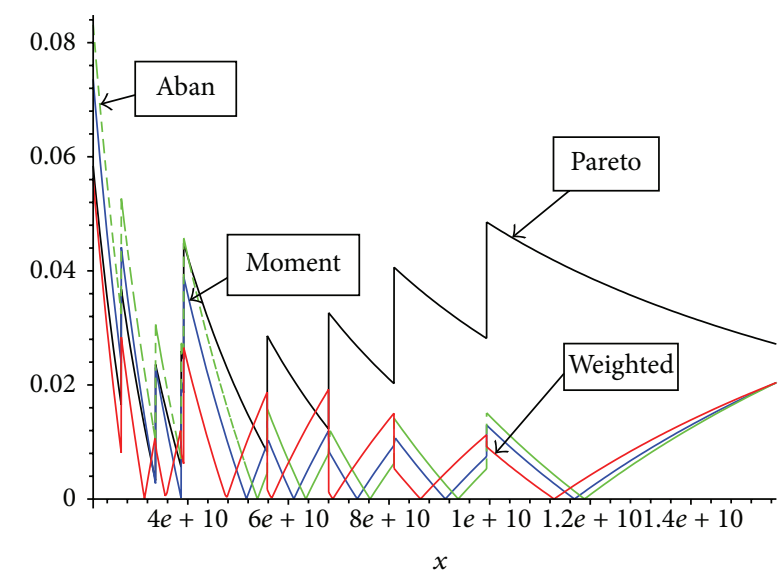

FIGURE 5: Absolute errors $\left|F^{*}(x)-S_{n}(x)\right|$ for hurricane loss example on the 10 largest losses data.

where $z$ is the observed value of $W_{n}^{2}$ and $\Gamma(x)=$ $\int_{0}^{\infty} t^{x-1} e^{-t} d t, x>0$, is the Gamma function.

(3) Cramer-von Mises test [16] proposed (C-v-M test) using $\psi(u)=1$ in (32); thus, under $H_{0}$, the test statistic and $P$-value are given by

$$
\begin{aligned}
n \omega^{2}=\frac{1}{12 n}+\sum_{j=1}^{n}\left(u_{j}-\frac{2 j-1}{2 n}\right)^{2}, \\
P \text {-value }=1-\frac{1}{\pi \sqrt{z}} \sum_{j=0}^{\infty}\left\{\frac{(-1)^{2 j} \Gamma(j+1 / 2)(4 j+1)^{1 / 2}}{j ! \sqrt{\pi}} e^{-(4 j+1)^{2} /(16 z)}\right. \\
\left.\times K_{1 / 4}\left(\frac{(4 j+1)^{2}}{16 z}\right)\right\},
\end{aligned}
$$

where $K_{1 / 4}(x)$ is the modified Bessel function of the second kind

$$
\begin{aligned}
& K_{\alpha}(x)=\frac{\pi}{2} \frac{I_{-\alpha}(x)-I_{\alpha}(x)}{\sin (\alpha \pi)}, \\
& I_{\alpha}(x)=\sum_{m=0}^{\infty} \frac{1}{m ! \Gamma(m+\alpha+1)}\left(\frac{x}{2}\right)^{2 m+\alpha} .
\end{aligned}
$$

For fitting the 49 losses in the hurricane loss data, for example, after computing estimates of $F^{*}(x)$ by using (26), (27), (28), and (29), we compute the absolute error (AE) in (30) and define the integrated error (IE) by

$$
\mathrm{IE}=\left[\int_{X_{(n-r+1)}}^{X_{(n)}}\left(S_{n}(x)-F^{*}(x)\right)^{2} d x\right]^{1 / 2} .
$$

Figure 5 gives the absolute errors (AE) in (30) for the 10 largest losses and confirms that the original Pareto estimate has relatively larger errors and the weighted estimate has relatively smaller errors in the tail. Figure 5 explains the data fitting of the tail of the distributions in Figure 4.

We also compute the AE in (30) and IE in (36) to confirm those tail errors by using $r$ as the number of the largest losses, $1 \leq r \leq n$. The AE and IE values are given in Table 4 (the smallest values are bold with $*$ ). 
TABLE 4: Errors of goodness-of-fit tests $n=49$ for hurricane example.

\begin{tabular}{|c|c|c|c|c|c|c|}
\hline \multirow{3}{*}{ Method } & \multicolumn{6}{|c|}{ Goodness-of-fit tests } \\
\hline & \multicolumn{3}{|c|}{ Absolute error (AE) } & \multicolumn{3}{|c|}{ Integrated error (IE) } \\
\hline & $r=49$ & $r=18$ & $r=10$ & $r=49$ & $r=18$ & $r=10$ \\
\hline Pareto $_{(\text {Hill })}$ & 0.1340 & 0.0584 & 0.0584 & 0.4844 & 0.3818 & 0.3723 \\
\hline $\mathrm{TPD}_{(\mathrm{Aban})}$ & $0.0948^{*}$ & 0.0839 & 0.0832 & 0.3114 & 0.2565 & 0.2161 \\
\hline $\mathrm{TPD}_{\text {(Moment) }}$ & 0.1053 & 0.0738 & 0.0737 & $0.2985^{*}$ & 0.2171 & 0.1825 \\
\hline $\mathrm{TPD}_{\text {(Weighted) }}$ & 0.1250 & $0.0561^{*}$ & $0.0561^{*}$ & 0.3098 & $0.1678^{*}$ & $0.1453^{*}$ \\
\hline
\end{tabular}

TABLE 5: The estimation of index, mean, median, and VaR for forest fire loss data.

\begin{tabular}{lcccc}
\hline Method & $\widehat{\alpha}$ & $\widehat{\mu}$ & Median & $5 \%$ value at risk \\
\hline Pareto $_{(\text {Hill) }}$ & 4.3044 & 1.096 billion & 1.09 billion & 1.855 billion \\
$\operatorname{TPD}_{\text {(Aban) }}$ & 0.7964 & 1.167 billion & 1.15 billion & 1.446 billion \\
$\operatorname{TPD}_{\text {(Moment) }}$ & 0.8260 & 1.166 billion & 1.15 billion & 1.446 billion \\
$\operatorname{TPD}_{\text {(Weighted) }}$ & 0.8642 & 1.165 billion & 1.15 billion & 1.446 billion \\
\hline
\end{tabular}

The weighted estimator has the smallest $\mathrm{AE}$ and IE values for $r=18$ and 10 largest losses, and its IE value is almost equal to the smallest IE value for $r=49$, all largest losses. We statistically conclude that the weighted estimated distribution is the best fit in the tail of the hurricane loss data.

6.2. Forest Fire Loss Data Example. Next we look at the forest fire loss example in Section 1.1.2. The data in Table 1 contains a relatively substantial number of large forest fire losses which convince us to use a truncated Pareto model and compare the four estimators in (26), (27), (28), and (29). We use the 25 largest losses in this study.

Figure 6 is a log-log plot which shows the upper tail for the forest fire loss data. The circles represent the real data, and the straight line represents the estimated original Pareto distribution. The dashed line, dotted line, and thick solid line represent the estimated truncated Pareto distribution by using Aban's, Moment, and the weighted estimators, respectively. We can see that the estimated truncated Pareto distributions fit the data very well using all three estimation methods and are much better than the original Pareto distribution. Around the tail, the weighted and the Moment estimation methods perform the best. The fact that the tail of the data curves downward in Figure 6 is the evidence in support of using a truncated Pareto model.

The results of these three estimators are listed in Table 5 by using the forest fire loss data, where $\hat{\gamma}=X_{(1)}, \widehat{v}=X_{(n)}$, and $n=25$.

Note that the weighted estimate $\widehat{\alpha}_{w}$ is the largest among the three truncated Pareto estimates. The 5\% value at risk of the original Pareto estimate is the largest. The 5\% VaR may be overestimated compared to the truncated Pareto models.

Similarly, as in the hurricane example, we compute the $\mathrm{AE}$ in (30) and IE in (36) again for fitting the 25 losses of the forest fire loss data.

Figure 7 gives the absolute errors (AE) in (30) for the 10 largest losses and confirms that the original Pareto estimate

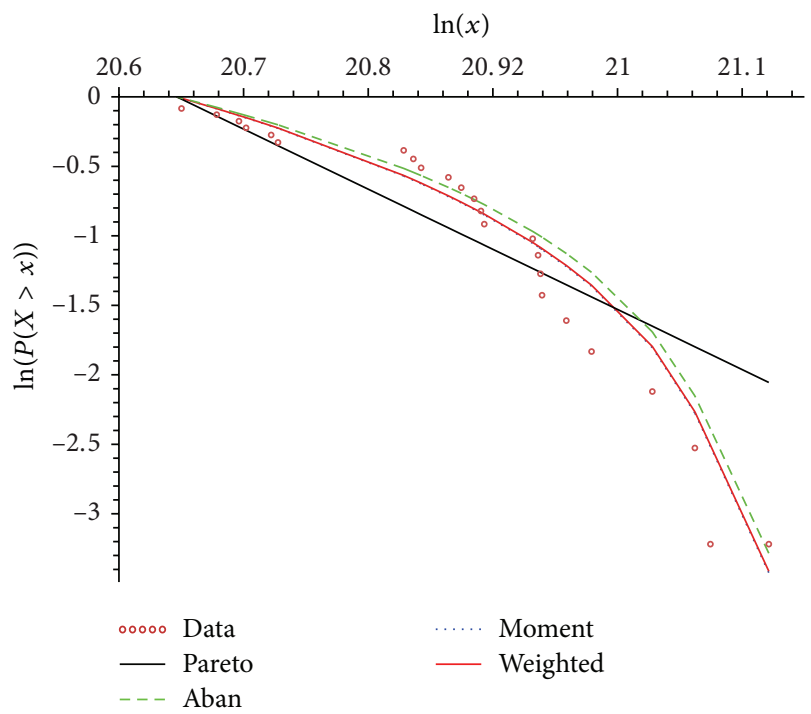

FIGURE 6: Log-log plot for forest fire example.

has relatively larger errors and the weighted estimate has relatively smaller errors in the tail. Figure 7 explains the data fitting of the tail of the distributions in Figure 6.

We also compute the AE in (30) and IE in (36) to confirm those tail errors by using $r$ as the number of the largest losses, $1 \leq r \leq n$. The IE values are given in Table 6 (the smallest values are bold with $*$ ).

The weighted estimator has the smallest $\mathrm{AE}$ and IE values for $r=10$ and 5, and its IE value in $r=25$ is almost equal to the smallest IE value. We statistically conclude that the weighted estimated distribution is the best fit to the tail of the forest fire loss data.

\section{Conclusions}

In the complicated real world, it is difficult to construct a model combining all the desired features. In general, the final model selection depends on the best fitting model. The criteria are based on goodness-of-fit tests, existence of the moments, characteristic largest values, and log-log plots. The hurricane loss data and forest fire loss data are well fitted by the truncated Pareto distribution. In summary,

(a) we recommend that the truncated Pareto model is appropriate as a loss distribution to be used when analyzing huge risk loss data sets. The upper and lower 
TABLE 6: Errors of goodness-of-fit tests $n=25$ for forest fire loss data.

\begin{tabular}{|c|c|c|c|c|c|c|}
\hline \multirow{3}{*}{ Method } & \multicolumn{6}{|c|}{ Goodness-of-fit tests } \\
\hline & \multicolumn{3}{|c|}{ Absolute error (AE) } & \multicolumn{3}{|c|}{ Integrated error (IE) } \\
\hline & $r=25$ & $r=10$ & $r=5$ & $r=25$ & $r=10$ & $r=5$ \\
\hline Pareto $_{(\text {Hill })}$ & 0.2656 & 0.1288 & 0.1288 & 2.7125 & 1.1493 & 1.0985 \\
\hline $\mathrm{TPD}_{(\mathrm{Aban})}$ & $0.1503^{*}$ & 0.1195 & 0.1195 & $1.3687^{*}$ & 0.7911 & 0.5430 \\
\hline $\mathrm{TPD}_{\text {(Moment) }}$ & 0.1520 & 0.0982 & 0.0982 & 1.3736 & 0.7764 & 0.5430 \\
\hline $\mathrm{TPD}_{\text {(Weighted) }}$ & 0.1542 & $0.0964^{*}$ & $0.0964^{*}$ & 1.3796 & $0.7577^{*}$ & $0.5203^{*}$ \\
\hline
\end{tabular}

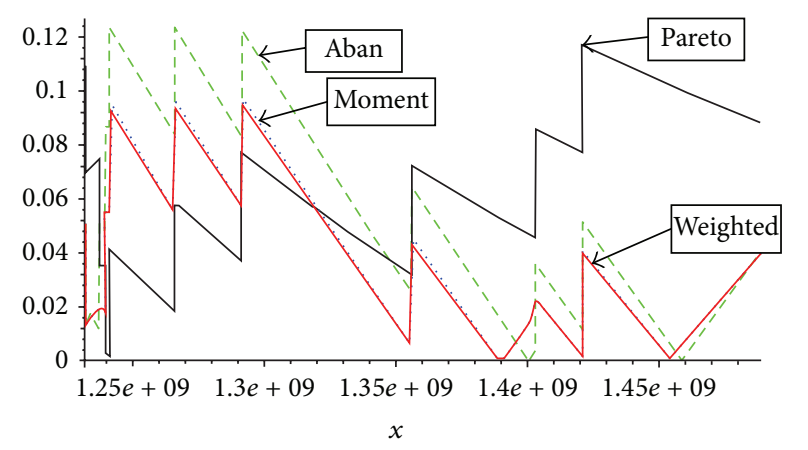

Figure 7: Absolute errors $\left|F^{*}(x)-S_{n}(x)\right|$ for forest fire loss example, the 10 largest losses.

limits can be set by the largest and smallest losses or other reasonable values;

(b) the estimated loss distribution provides a prediction of the next disaster's $5 \%$ value at risk. The largest loss in the data set plays an important role in the prediction. In Figures 4 and 6 , the trend of the tail distribution is crucial for an insurance company setting policy, also for inhabitants and government to make plans to minimize damage from natural disasters;

(c) the semiparametric methods (Moment and weighted) are robust, easy to use, more stable, and fit the data better than the MLE methods. In both of the foregoing two examples, the estimated shape parameter $\widehat{\alpha}$ is less than 1. The original Pareto model is not able to use the Moment or the weighted methods. This is another advantage of using the truncated Pareto model;

(d) the statistical inference in these examples shows that the estimated distribution curve by using proposed weighted estimation method fits the tails of data better, relative to the Moment and MLE estimators. This is due to the fact that the weighted estimator gives less weight on the extreme values and has good MSE. Based on these studies, we suggest that further studies on the usage of weights may be useful.

\section{Appendix}

\section{Mathematical Derivations}

Lemma A.1. For a truncated Pareto random variable $X$ with p.d.f. given in (3), for $i, j \in\{1,2, \ldots, n\}, i<j, X_{(1)} \leq X_{(2)} \leq$ $\cdots \leq X_{(n)}$ are order statistics; one has

$$
\begin{gathered}
E\left[X_{(i)}\right]=\gamma \sum_{k=0}^{\infty}\left(\begin{array}{c}
\frac{1}{\alpha}+k-1 \\
k
\end{array}\right) \rho^{k} \frac{\Gamma(n+1) \Gamma(k+i)}{\Gamma(i) \Gamma(k+n+1)}, \\
\left.E\left[X_{(i)}^{2}\right]=\gamma \sum_{k=0}^{\infty}\left(\begin{array}{c}
\frac{2}{\alpha}+k-1 \\
k
\end{array}\right) \rho^{k} \frac{\Gamma(n+1) \Gamma(k+i)}{\Gamma(i) \Gamma(k+n+1)}, \quad \text { A. } 2\right) \\
E\left[X_{(i)} X_{(j)}\right] \\
=\gamma^{2} \sum_{k=0}^{\infty} \sum_{l=0}^{\infty}\left\{\left(\begin{array}{c}
\frac{1}{\alpha}+k-1 \\
k
\end{array}\right)\left(\frac{1}{\alpha}+l-1\right)\right. \\
\left.\quad \times \rho^{k+l}\left[\frac{\Gamma(n+1) \Gamma(k+i) \Gamma(k+l+j)}{\Gamma(i) \Gamma(k+j) \Gamma(k+l+n+1)}\right]\right\}
\end{gathered}
$$

where $\rho=\left(g^{\alpha}-1\right) / g^{\alpha}, g=\nu / \gamma, 0<\rho<1,0<g<1$, $0<\gamma \leq x \leq \nu<\infty$, and $\alpha>0$.

Proof. Let the c.d.f. of a truncated Pareto distribution in (4) be

$$
\begin{aligned}
& F(x)=u, \quad x=\gamma(1-b u)^{-1 / \alpha}, \\
& \rho=\frac{g^{\alpha}-1}{g^{\alpha}}, \quad g=\frac{\nu}{\gamma} .
\end{aligned}
$$

By the theory of order statistics, we have

$$
\begin{aligned}
& f_{i}(x)=\frac{\Gamma(n+1)}{\Gamma(i) \Gamma(n-i+1)}[F(x)]^{i-1}[1-F(x)]^{n-i} f(x) \\
& f_{i, j}(x, y)= \frac{\Gamma(n+1)}{\Gamma(i) \Gamma(j-i) \Gamma(n-j+1)} \\
& \times[F(x)]^{i-1}[F(y)-F(x)]^{j-i-1} \\
& \times[1-F(y)]^{n-j} f(x) f(y) .
\end{aligned}
$$


Using the binomial formula $1 /(1-x)^{r}=\sum_{k=0}^{\infty}\left(\begin{array}{c}r+k-1 \\ k\end{array}\right) x^{k}$, $m=1,2, \ldots$, we have

$$
\begin{aligned}
& E\left[X_{(i)}^{m}\right]= \int_{\gamma}^{\nu} x^{m} f_{i}(x) d x \\
&= \int_{0}^{1}\left\{\gamma(1-\rho u)^{-m / \alpha} \frac{\Gamma(n+1)}{\Gamma(i) \Gamma(n-i+1)}\right. \\
&\left.\times(u)^{i-1}(1-u)^{n-i} d u\right\} \\
&= \frac{\Gamma(n+1)}{\Gamma(i) \Gamma(n-i+1)} \gamma \\
& \times \sum_{k=0}^{\infty}\left\{\left(\frac{m}{\alpha}+k-1\right)\right. \\
&= \gamma \sum_{k=0}^{\infty}\left(\begin{array}{c}
\frac{m}{\alpha}+k-1 \\
k
\end{array}\right) \rho^{k} \frac{\Gamma(n+1) \Gamma(k+i)}{\Gamma(i) \Gamma(k+n+1)} \\
&\left.\times \int_{0}^{1} \rho^{k}(u)^{k+i-1}(1-u)^{n-i} d u\right\}
\end{aligned}
$$

By substituting $m=1$ and 2, respectively, we have (A.1) and (A.2). And

$$
\begin{aligned}
E\left[X_{(i)} X_{(j)}\right] & \\
= & \int_{\gamma}^{y} \int_{\gamma}^{\nu} x_{i} x_{j} f_{i, j}(x, y) d x d y \\
= & \frac{\Gamma(n+1)}{\Gamma(i) \Gamma(j-i) \Gamma(n-j+1)} \\
& \times \int_{0}^{1} \int_{0}^{1}\left\{\gamma(1-\rho u)^{-1 / \alpha} \gamma(1-\rho h)^{-1 / \alpha}\right. \\
= & \frac{\left.\Gamma(u)^{i-1}(h-u)^{j-i-1}(1-h)^{h-j} d u d h\right\}}{\Gamma(i) \Gamma(j-i) \Gamma(n-j+1)} \gamma^{2} \\
& \times \sum_{k=0}^{\infty} \sum_{k=0}^{\infty}\left\{\left(\frac{1}{\alpha}+k-1\right)\left(\frac{1}{\alpha}+l-1\right)\right. \\
& \times \rho^{k+l} \int_{0}^{1} \int_{0}^{1}\left(\frac{u}{h}\right)^{k+i-1}\left(1-\frac{u}{h}\right)^{j-i-1}
\end{aligned}
$$

$$
\begin{aligned}
=\sum_{k=0}^{\infty} \sum_{l=0}^{\infty}\{ & \left(\begin{array}{c}
\frac{1}{\alpha}+k-1 \\
k
\end{array}\right)\left(\begin{array}{c}
\frac{1}{\alpha}+l-1 \\
l
\end{array}\right) \\
& \left.\times \rho^{k+l} \frac{\Gamma(n+1) \Gamma(k+i) \Gamma(k+l+j)}{\Gamma(i) \Gamma(k+j) \Gamma(k+l+n+1)}\right\} .
\end{aligned}
$$

Proof of Theorem 3. When $\alpha>0$, we use

$$
\begin{aligned}
E\left[\widehat{\mu}_{w}\right]=\sum_{i=2}^{n-1}\left\{E\left[X_{(i)}\right]+\frac{1}{2}(1-(n-2) w)\right. & \\
& \left.\times\left(E\left[X_{(1)}\right]+E\left[X_{(n)}\right]\right)\right\}, \\
\operatorname{MSE}\left(\widehat{\mu}_{w}\right)= & E\left[\left(\widehat{\mu}_{w}-\mu\right)^{2}\right] \\
= & E\left[\widehat{\mu}_{w}^{2}\right]-2 \mu E\left[\widehat{\mu}_{w}\right]+\mu^{2} .
\end{aligned}
$$

And we have

$$
\begin{aligned}
E\left[\widehat{\mu}_{w}^{2}\right]= & \sum_{i=2}^{n-1} w^{2} E\left[X_{(i)}^{2}\right]+2 \sum_{i=2}^{n-2} \sum_{j=i+1}^{n-1} w^{2} E\left[X_{(i)} X_{(j)}\right] \\
& +\frac{1}{4}(1-(n-2) w)^{2} \\
& \times\left(E\left[X_{(1)}^{2}\right]+E\left[X_{(n)}^{2}\right]+2 E\left[X_{(1)} X_{(n)}\right]\right) \\
& +\sum_{i=2}^{n-1}\{w(1-(n-2) w) \\
& \left.\times\left(E\left[X_{(1)} X_{(i)}\right]+E\left[X_{(i)} X_{(n)}\right]\right)\right\}
\end{aligned}
$$

Let

$$
\begin{gathered}
A=\sum_{i=2}^{n-1} E\left[X_{(i)}^{2}\right]+2 \sum_{i=2}^{n-2} \sum_{j=i+1}^{n-1} E\left[X_{(i)} X_{(j)}\right] \\
B=E\left[X_{(1)}^{2}\right]+E\left[X_{(n)}^{2}\right]+2 E\left[X_{(1)} X_{(n)}\right] \\
C=\sum_{i=2}^{n-1}\left(E\left[X_{(1)} X_{(i)}\right]+E\left[X_{(i)} X_{(n)}\right]\right), \\
D=\sum_{i=2}^{n-1} E\left[X_{(i)}\right] \\
E=E\left[X_{(1)}\right]+E\left[X_{(n)}\right] .
\end{gathered}
$$

Then

$$
\begin{aligned}
\operatorname{MSE}\left(\widehat{\mu}_{w}\right)= & A w^{2}+\frac{1}{4}(1-(n-2) w)^{2} B \\
& +w(1-(n-2) w) C \\
& -2 \mu\left(D w+\frac{1}{2}(1-(n-2) w) E\right)+\mu^{2}
\end{aligned}
$$




$$
\begin{aligned}
= & \left(A+\frac{1}{4}(n-2)^{2} B-(n-2) C\right) w^{2} \\
& +\left(-\frac{1}{2}(n-2) B+C-2 \mu D+\mu(n-2) E\right) w \\
& +\frac{1}{4} B-\mu E+\mu^{2} .
\end{aligned}
$$

Now, use Lemma A.1; then we have Theorem 3.

Lemma A.2. $\operatorname{MSE}\left(\widehat{\mu}_{w}\right)$ in (20) is a convex function with $a>0$; when $n>4 C / B+2, C$ and $B$ are given in (20).

Proof. Let $f(w)=\operatorname{MSE}\left(\widehat{\mu}_{w}\right)=a w^{2}+b w+c$. We have

$$
a=A+\frac{1}{4}(n-2)^{2} B-(n-2) C
$$

where

$$
\begin{gathered}
A=E\left[\left(\sum_{i=2}^{n-1} X_{(i)}\right)^{2}\right] \geq 0, \\
B=E\left[\left(X_{(1)}+X_{(n)}\right)^{2}\right] \geq 0, \\
C=E\left[\sum_{i=2}^{n-1} X_{(i)}\left(X_{(1)}+X_{(n)}\right)\right] \geq 0 .
\end{gathered}
$$

Since $n>2$, then

$\frac{1}{4}(n-2) B-C>0, \quad$ such that $a>0$, when $n>\frac{4 C}{B}+2$.

Proof of Corollary 5. By Theorem 3 and Lemma A.2, let

$$
f(w)=\operatorname{MSE}\left(\widehat{\mu}_{w}\right)=a w^{2}+b w+c,
$$

which is a quadratic function with first and second derivatives of $f(w)$ w.r.t. $w$ :

$$
f^{\prime}(w)=2 a w+b, \quad f^{\prime \prime}(w)=2 a>0 .
$$

Hence, $f(w)$ is a convex function with minimum value

$$
f_{\min }=a\left(-\frac{b}{2 a}\right)^{2}+b\left(-\frac{b}{2 a}\right)+c=-\frac{b^{2}}{4 a}+c .
$$

The maximum value of the $\operatorname{EFF}\left(\widehat{\mu}_{w}\right)$ is

$$
\begin{aligned}
& \operatorname{EFF}_{\text {max }}\left(\widehat{\mu}_{w}\right) \\
& =\frac{\alpha \gamma^{\alpha}\left(\nu^{2-\alpha}-\gamma^{2-\alpha}\right) /\left\{(2-\alpha)\left(1-(\gamma / \nu)^{\alpha}\right)\right\}}{n\left(-b^{2} /(4 a)+c\right)} \\
& -\frac{\alpha^{2} \gamma^{2 \alpha}\left(\nu^{1-\alpha}-\gamma^{1-\alpha}\right)^{2} /\left\{(1-\alpha)^{2}\left(1-(\gamma / \nu)^{\alpha}\right)^{2}\right\}}{n\left(-b^{2} /(4 a)+c\right)} \\
& \text { at } w=-\frac{b}{2 a} .
\end{aligned}
$$

\section{Acknowledgments}

The authors thank the referees and the editor for their comments which helped to improve the paper. This research is supported by the Natural Sciences and Engineering Research Council of Canada.

\section{References}

[1] R. A. Pielke Jr., J. Gratz, C. W. Landsea, D. Collins, M. A. Saunders, and R. Musulin, "Normalized hurricane damage in the United States: 1900-2005," Natural Hazards Review, vol. 9, no. 1, pp. 29-42, 2008.

[2] P. Embrechts, C. Klüppelberg, and T. Mikosch, Modelling Extremal Events for Insurance and Finance, Springer, New York, NY, USA, 2003.

[3] C. Kleiber and S. Kotz, Statistical Size Distribution in Economics and Actuarial Sciences, John Wiley \& Sons, New York, NY, USA, 2003.

[4] Beirlant, J. Y. Goegebeur, I. Sergers, and J. Teugels, Statistics of Extremes, Theory and Application, John Wiley \& Sons, New York, NY, USA, 2005.

[5] M. A. Beg, "Estimation of the tail probability of the truncated Pareto distribution," Journal of Information \& Optimization Sciences, vol. 2, no. 2, pp. 192-198, 1981.

[6] I. B. Aban, M. M. Meerschaert, and A. K. Panorska, "Parameter estimation for the truncated Pareto distribution," Journal of the American Statistical Association, vol. 101, no. 473, pp. 270-277, 2006.

[7] B. Efron, "Bootstrap methods: another look at the jackknife," The Annals of Statistics, vol. 7, no. 1, pp. 1-26, 1979.

[8] P. Barbe and P. Bertail, The Weighted Bootstrap, Springer, New York, NY, USA, 1995.

[9] G. R. Shorack and J. A. Wellner, Empirical Processes with Applications to Statistics, John Wiley \& Sons, New York, NY, USA, 1986.

[10] H. L. Koul, Weighted Empirical and Linear Models, vol. 21 of Lecture Notes-Monograph Series, Institute of Mathematical Statistics, Hayward, Calif, USA, 1992.

[11] M. L. Huang and P. H. Brill, "A distribution estimation method based on level crossings," Journal of Statistical Planning and Inference, vol. 124, no. 1, pp. 45-62, 2004.

[12] M. L. Huang, "The efficiencies of a weighted distribution function estimator," in The Proceeding of American Statistical Association, Nonparametric Statistics Section, pp. 1502-1506, 2003.

[13] B. M. Hill, "A simple general approach to inference about the tail of a distribution," The Annals of Statistics, vol. 3, no. 5, pp. 1163-1174, 1975.

[14] P. Cooke, "Statistical inference for bounds of random variables," Biometrika, vol. 66, no. 2, pp. 367-374, 1979.

[15] A. N. Kolmogorov, "Sulla determinazione empirica di una legge di distribuzione," Giornale dell'Istituto Italiano degli Attuari, vol. 4, pp. 83-91, 1933.

[16] T. W. Anderson and D. A. Darling, "Asymptotic theory of certain "goodness of fit" criteria based on stochastic processes," The Annals of Mathematical Statistics, vol. 23, pp. 193-212, 1952. 


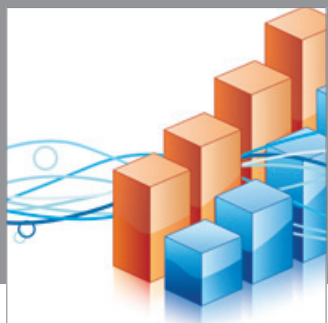

Advances in

Operations Research

mansans

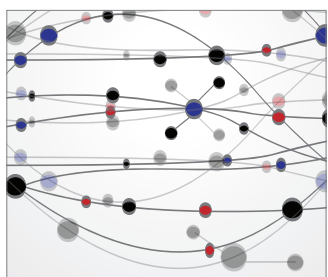

The Scientific World Journal
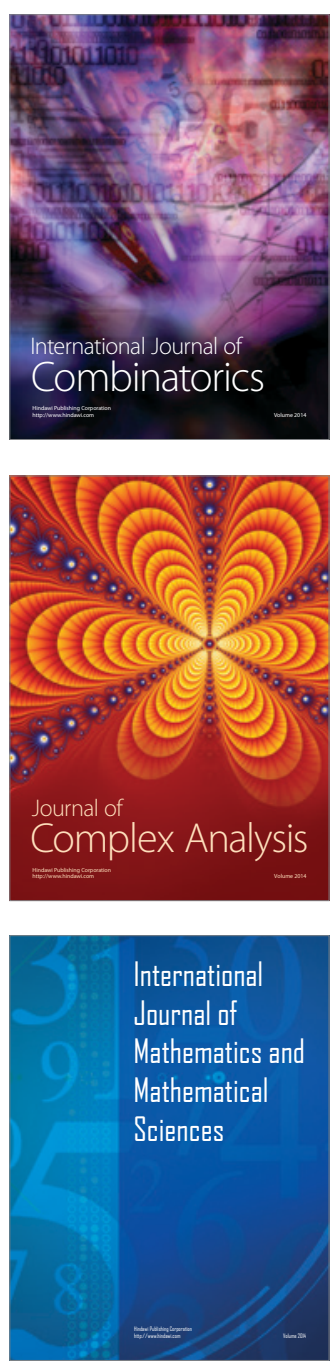
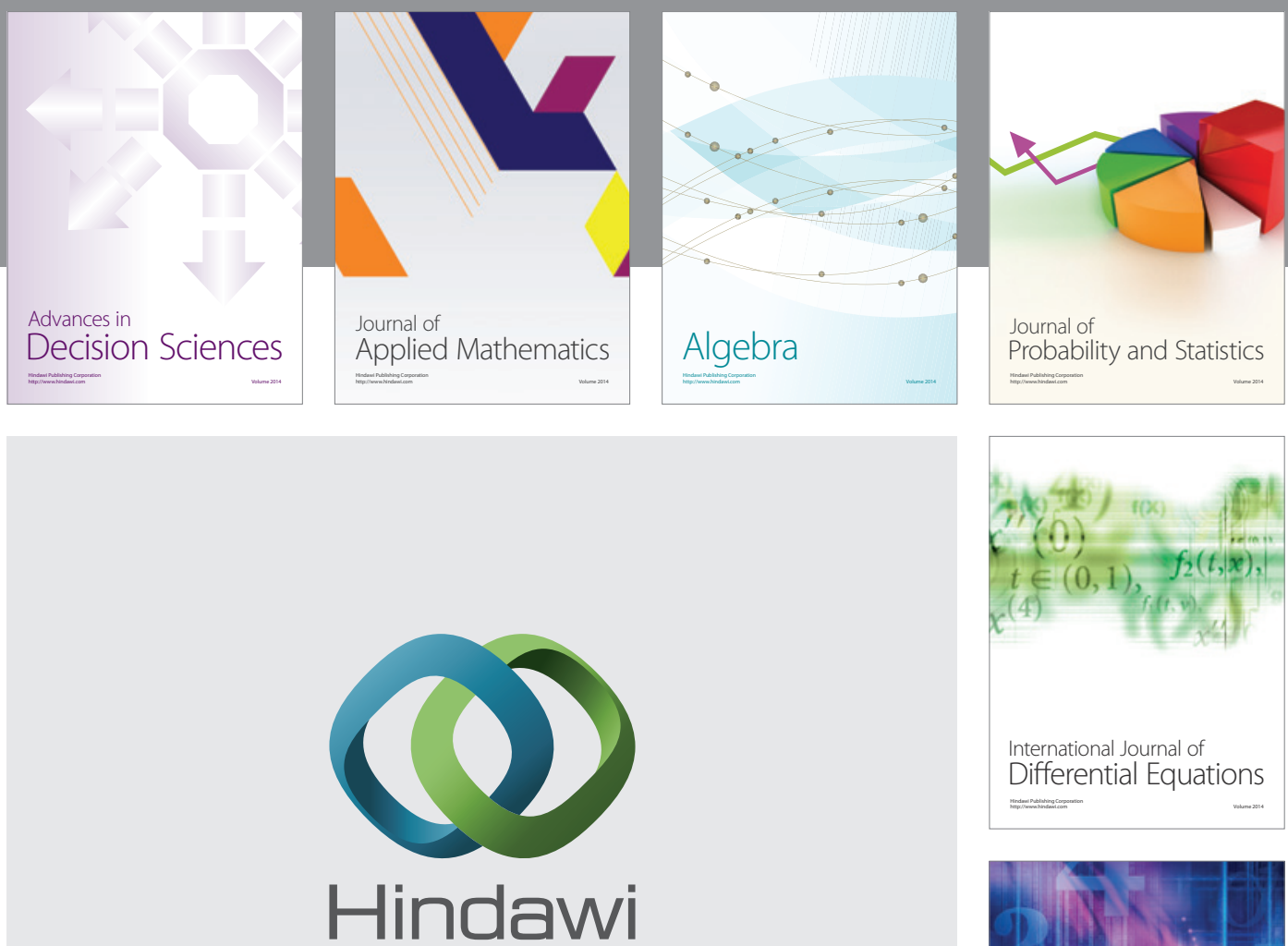

Submit your manuscripts at http://www.hindawi.com
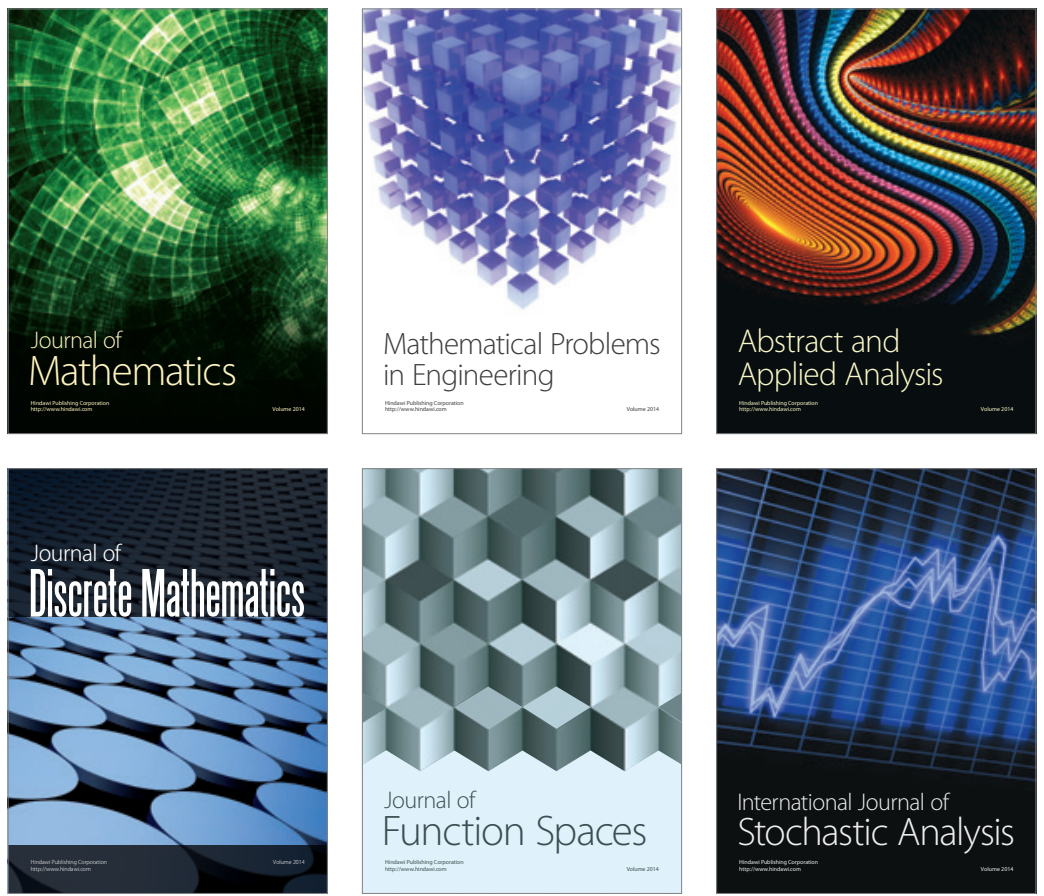

Journal of

Function Spaces

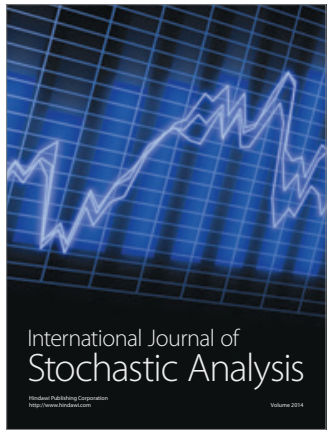

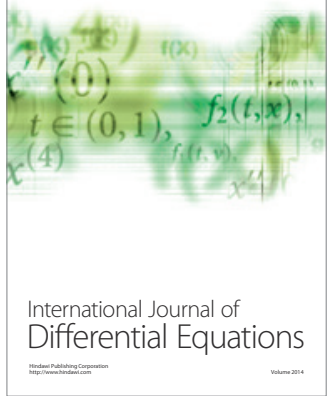
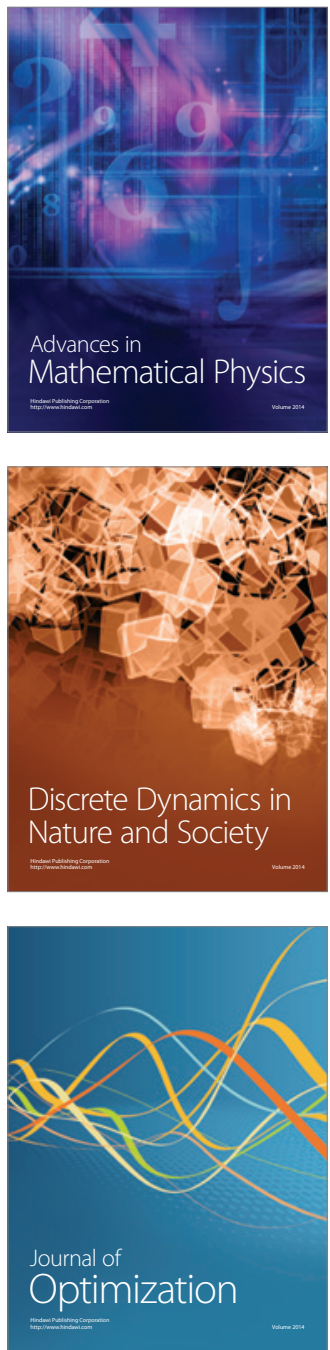